\title{
Miniaturisierung des Wachstumshemmtestes mit der Grünalge Desmodesmus subspicatus nach DIN 38412 Teil 33
}

\author{
Alexander Liebenstein • Werner Manz • Roland Pesch • \\ Gunther Schmidt · Winfried Schröder · Dierk-Steffen Wahrendorf
}

Erhalten: 23. Juli 2008/Akzeptiert: 24. März 2009/Online veröffentlicht: 25. August 2009

(C) Springer-Verlag 2009

\begin{abstract}
Zusammenfassung Hintergrund, Ziel und Zweck Die ökotoxikologische Untersuchung von limnischen Sedimenten und Baggergut sowie die Abschätzung des Belastungspotenzials erfolgt gemäß den Handlungsanweisungen für den Umgang mit Baggergut der Wasser- und Schifffahrtsverwaltung des Bundes (HABAB-WSV) im Standardprüfverfahren mit genormten Biotests. Von den zu untersuchenden Sedimenten werden Porenwässer und Eluate gewonnen und über Verdünnungsreihen mit Organismen unterschiedlicher trophischer Ebenen untersucht. Im Merkblatt „Ökotoxikologische Baggergutuntersuchungen“" (BfG 2007) ist zur Untersuchung von Sedimenten des Binnenbereiches neben weiteren Testverfahren der Wachstumshemmtest mit der Grünalge Desmodesmus subspicatus nach DIN 38412 Teil 33 (1991) angeführt.
\end{abstract}

Der Grünalgentest wird in den Laboratorien der Bundesanstalt für Gewässerkunde (BfG) entsprechend den Normvorgaben in Kulturröhrchen aus Glas (Reagenzglasvolumen $10 \mathrm{ml}$ ) durchgeführt. Im Folgenden wird diese Methodendurchführung als herkömmliche Methode oder als DINMethode bezeichnet.

Im Rahmen einer Diplomarbeit sollte an der BfG ein Verfahren zur Durchführung des Algentests in Mikrotiterplatten entwickelt werden, das zur DIN-Methode vergleichbare Ergebnisse liefert. Durch die Miniaturisierung und eine Automatisierung des Algentests sollten Messkapazität und

Verantwortliche Herausgeberin: Karen Duis

A. Liebenstein $(\varangle) \cdot$ R. Pesch $\cdot$ G. Schmidt $\cdot$ W. Schröder Hochschule Vechta, Lehrstuhl für Landschaftsökologie, Eichendorffweg 30, 49377 Vechta, Deutschland

E-Mail: aliebenstein@gmail.com

W. Manz $\cdot$ D.-S. Wahrendorf

Bundesanstalt für Gewässerkunde,

Am Mainzer Tor 1, 56068 Koblenz, Deutschland
Probendurchsatz erhöht und im Gegenzug der Arbeitszeitund Personalaufwand reduziert werden.

Methoden Grundlage für die miniaturisierte Testdurchführung war der Algentest nach DIN 38412 Teil 33. Die Methodendurchführung in Mikrotiterplatten wurde durch systematische Untersuchungen optimiert. So wurden z.B. geeignete Kultivierungsbedingungen wie Abdeckung und Art der Reaktionskammern ermittelt und die Parametereinstellungen des Messgerätes optimiert. Zur Validierung der mit der entwickelten miniaturisierten Methode gewonnenen Ergebnisse wurden 25 Testgüter (20 Porenwässer und 5 Einzelsubstanzen) untersucht und mit den Messergebnissen der herkömmlichen Methode nach DIN verglichen.

Ergebnisse Der Vergleich der parallel durchgeführten Methoden in der Mikrotiterplatte und im Reagenzglas zeigte eine durchschnittliche Abweichung der Hemmwirkungen von ca. 20 Prozentpunkten. Eine statistische Überprüfung mit dem Mann-Whitney-U-Test bezüglich der Gleichheit der in den Testkonzentrationen festgestellten Hemmwirkungen zeigte für ca. $30 \%$ der untersuchten Proben eine Übereinstimmung. Mit den über die Verdünnungsreihen ermittelten Toxizitäten wurde der pT-Wert bestimmt (Krebs 1988). Bei zehn der 20 mit beiden Methoden untersuchten Umweltproben wurde derselbe pT-Wert ermittelt (50\%). Bei $40 \%$ der untersuchten Sedimente wurde eine Abweichung um einen pT-Wert und bei $10 \%$ eine Abweichung um mehr als einen pT-Wert festgestellt.

Diskussion Bei der Optimierung der Methodendurchführung wurden grundlegende Parameter untersucht und an das miniaturisierte System angepasst, um möglichst alle der in DIN 38412 Teil 33 geforderten Kriterien einzuhalten. Als problematisch erwies sich hierbei das in der Norm geforderte Gültigkeitskriterium zur Biomasseerhöhung im Kontrollansatz um den Faktor 30, das nur in zwei von 31 Versuchsdurchführungen erfüllt wurde. 
Die auf einem Vergleich mit der herkömmlichen Methode nach DIN beruhende Validierung der in den Verdünnungsstufen festgestellten Hemmwirkungen ergab teilweise statistisch stark signifikante Unterschiede. Insgesamt lagen für ca. zwei Drittel der festgestellten Hemmwirkungen signifikante Unterschiede zwischen beiden Methoden vor.

Schlussfolgerungen Ohne eine weitere Anpassung und Optimierung kann die hier entwickelte miniaturisierte Methodendurchführung für den Algentest die herkömmliche Durchführung nicht ersetzen.

Empfehlungen und Ausblick Zur miniaturisierten Durchführung des Algentestes in Mikrotiterplatten sollten weiterführende Untersuchungen und Optimierungen erfolgen. Die Gültigkeitskriterien der Norm wie z.B. die Vervielfachung der Biomasse im Kontrollansatz sollten zuverlässig eingehalten werden können. Eine miniaturisierte Methodendurchführung des Algentestes ist weiterhin grundsätzlich anzustreben, um die Kapazität zu steigern und um gleichzeitig den Arbeitsaufwand durch automatisierte Arbeitsschritte zu verringern. Die mit der miniaturisierten Methode erzeugten Ergebnisse sollten denen der bislang durchgeführten Methode entsprechen.

Schlüsselwörter Algentest · Biotest · Chlorophyll-Fluoreszenz · Desmodesmus subspicatus · DIN 38412 Teil 33 . Mikrotiterplatte $\cdot$ Miniaturisierung $\cdot$ Wachstumshemmtest

\section{Miniaturization of the growth inhibition test with the green algae Desmodesmus subspicatus according to DIN 38412-33}

\begin{abstract}
Background, aim, and scope Biological test systems have been successfully used to analyse and assess the ecotoxicological potentials of sediments and dredged materials. In compliance with the German directive for dredgedmaterial management (HABAB-WSV, BfG 2000) standardised bioassays with organisms of different trophic levels are applied to limnic sediments. The growth-inhibition test with the green alga Desmodesmus subspicatus according to DIN 38412 part 33 is one of three mandatory bioassays (Guidance paper "Ökotoxikologische Baggergutuntersuchungen", BfG 2007).

The laboratories of the German Federal Institute of Hydrology $(B f G)$ conduct this algal test in agreement with the DIN standard in test tubes of $10 \mathrm{ml}$ volume. In the following, this method is called the "conventional method" or "DIN-method".

The research described below was conducted in the $B f G$ in a diploma-thesis aiming to develop a miniaturized algae test procedure that uses microtiter plates, but yields comparable results. Automation and miniaturization should
\end{abstract}

increase the throughput capacity and reduce the time and labour requirements.

Materials and methods The growth-inhibition test with the green alga Desmodesmus subspicatus according to DIN standard 38412 part 33 was taken as the starting point for the miniaturization. The testing method in microtiter plates was optimised by systematic examination of the cultivation conditions, coverages of the wells, and settings of the measuring instruments. To verify the results of the miniaturized test procedure, 25 environmental samples ( 20 pore waters and five single substances) were tested with the conventional and the miniaturized procedure. The results of both test procedures were compared using statistical analyses.

Results The comparison of the growth inhibition test results from the microtiter plates with those from the conventional procedure indicated an average deviation of about $20 \%$-points. In approximately $30 \%$ of the cases, a statistical verification with the Mann-Whitney-U-Test showed agreement of the inhibition values obtained with both methods. A method to express the ecotoxicological potential of a sample is the pT-value (Krebs 1988). The pT-values were determined based on the toxicities obtained using serial dilution. (Krebs 1988). When comparing both methods 10 out of 20 environmental samples had the same pT-value (50\%). In $40 \%$ of the samples, the deviation was one pT-value, in $10 \%$ it was more than one pT-value.

Discussion During the optimization of the method, different parameters of the algal growth inhibition test were examined and adapted to the miniaturized system, so that all criteria required after DIN 38412 part 33 should be met. However, the miniaturized test did not reliably achieve these validity criteria, namely the increase in the biomass by a factor of 30 in the controls. This criterion was only fulfilled in two out of 31 test runs.

The validation of the miniaturized growth inhibition test on the basis of a statistical comparison with the conventional method showed partially highly significant differences. Overall, significantly different inhibition values were found in about $60 \%$ of the cases.

Conclusions Without further adjustments and optimization it is not possible to substitute the conventional method by the miniaturized system.

Recommendations and perspectives Further research is required to miniaturize the test procedure and to perform the algae test on microtiter plates. The validity criteria of the DIN standard, such as the increase of biomass production in the controls, should be reliably met. Basically, the aim of miniaturizing the algae test procedure is the increase in throughput capacity and, at the same time, reduced work efforts by automation. However, the test results of the miniaturized method should be comparable to those of the conventional method. 
Keywords Algae Test $\cdot$ Bioassay $\cdot$ Biological Test . Chlorophyll-fluorescence · Desmodesmus subspicatus . DIN 38412 part 33 . Growth inhibition test . Microtiter plate $\cdot$ Miniaturization

\section{Einleitung}

Aus vielen Quellen gelangen Schadstoffe wie Schwermetallverbindungen, Pestizide und halogenierte Kohlenwasserstoffe in Gewässer (Cleuvers 2002; Fent 2003). Diese können sich an Schwebstoffe und Sedimente anlagern und durch Veränderungen der hydrophysikalischen, chemischen oder biologischen Bedingungen remobilisiert werden (Ahlf 1995; Fomin et al. 2003). Die im Poren- oder Interstitialwasser gelösten Umweltchemikalien sind z. B. für benthische Organismen sehr gut verfügbar (Ahlf et al. 2001; Fent 2003), die über die Nahrungskette auch mit den anderen trophischen Ebenen des aquatischen Ökosystems interagieren. Remobilisierte Schadstoffe können das Ökosystem Fließgewässer erheblich belasten (Förstner 2000).

Im Zuge von Unterhaltungs-, Ausbau- und Neubaumaßnahmen im Bereich von Schifffahrtswegen, Häfen oder zur Gewährleistung des Abflussprofils werden Sedimente dem Gewässer entnommen oder relokalisiert (Henschel et al. 2003). Ist es vorgesehen, Baggergut innerhalb eines Gewässers umzulagern, so soll die Baggergutbeschaffenheit vor einer Umlagerung zur Beachtung ökologischer Belange gemäß HABAB-WSV (2000) geprüft werden. Diese Prüfung erfolgt anhand physikalischer, chemischer, biochemischer und ökotoxikologischer Kriterien (HABAB-WSV 2000).

Zur Beurteilung des ökotoxikologischen Belastungspotenzials von Baggergut und Sedimenten werden Biotestsysteme eingesetzt. Das Verfahren, mit dem limnisches Probenmaterial auf der trophischen Ebene der Produzenten auf die ökotoxikologische Schadwirkung getestet wird, ist der Algentest mit der Grünalge Desmodesmus subspicatus nach DIN 38412 Teil 33 (HABAB-WSV 2000). Die Untersuchung von Sedimenten mit dem Algentest erfolgt im Regelfall am Porenwasser und am Eluat. Für die Testreihe nach dem standardisierten Verfahren werden Verdünnungsreihen in geometrischer Folge hergestellt, mit denen nach der Schwellenkonzentration gesucht wird, bei der keine Schadwirkung mehr feststellbar ist (Krebs 2001). Relevant für die Aussage des Tests ist die Hemmung der Biomasseproduktion. Diese wird anhand des Algenwachstums in den Verdünnungsstufen im Vergleich zum Wachstum der Kontrollen definiert und über die Chlorophyll-Fluoreszenz ermittelt (Ahlf 1995; DIN 38412 Teil 33 1991; DIN EN ISO 8692 2004). Die erste Verdünnungsstufe, deren Hemmwirkung auf die Biomasseproduktion $<20 \%$ ist, dient zur Charakterisierung der Toxizität eines Testguts (Krebs 2000). Zur zahlenmäßigen Bezeichnung der ermittelten Toxizität wird der negative binäre Logarithmus der ersten nicht mehr toxischen Verdünnungsstufe in einer Verdünnungsreihe mit dem Verdünnungsfaktor 2 verwendet. Dieser als pT-Wert bezeichnete Faktor gibt an, wie oft eine Probe im Verhältnis 1:2 verdünnt werden muss, damit sie nicht mehr toxisch wirkt (Krebs 1988, 2005).

In den vergangenen zwanzig Jahren hat das Interesse an miniaturisierten Testsystemen deutlich zugenommen (Radetski et al. 1995). Viele miniaturisierte Testsysteme wurden bereits getestet (siehe z. B. Paixão et al. 2008; Pavlić et al. 2006). Die Bundesanstalt für Gewässerkunde befasst sich ebenfalls seit längerem mit der Miniaturisierung von Analysenverfahren.

Ziel der Arbeit war die Miniaturisierung der bestehenden Methodendurchführung, sodass der Algentest in Mikrotiterplatten durchgeführt werden kann. Dadurch wird ein höherer Probendurchsatz ermöglicht und darüber hinaus ist eine höhere Zahl an Replikaten realisierbar. Da die Testansätze direkt in den Wells der Mikrotiterplatte ausgemessen werden, ist es ohne zusätzlichen Aufwand möglich, Reaktionskinetiken zu erfassen. Die Aufnahme dieser Daten ermöglicht eine Ergänzung der bislang üblichen Endpunkte durch Integralanalysen (Schmitz et al. 1999). Für die angestrebte Miniaturisierung war es notwendig, die Messmethodik, die Inkubation etc. dem Verfahren anzupassen. Das Ergebnis der Untersuchungen sollte eine entsprechend angepasste Versuchsmethodik sein. Die Überprüfung der Validität der Messergebnisse der miniaturisierten Methode im Vergleich zur herkömmlichen Methode erfolgte durch statistische Untersuchungen. Die Ergebnisse sollten nicht signifikant von denen des Normverfahrens (DIN-Methode) abweichen und dessen Empfindlichkeit und Genauigkeit erzielen (Schmitz et al. 1999).

\section{Material und Methoden}

Im Rahmen der Untersuchungen zur Entwicklung und Optimierung sowie zur Validierung der miniaturisierten Methode wurde der Wachstumshemmtest mit der Grünalge Desmodesmus subspicatus in Anlehnung an DIN 38412 Teil 33 (1991) und unter Berücksichtigung des AQS-Merkblatts P-9/3 (1998) durchgeführt.

Zur Quantifizierung der Biomasseproduktion wurde die Chlorophyll-Fluoreszenz der Testansätze in der Mikrotiterplatte mit einem Fluoreszenz-Spektrofotometer (TECAN Safire, TECAN GmbH, Crailsheim) gemessen. Die Einstellungen der Messparameter wurden optimiert. Die Einstellung, die zu einem kleineren Variationskoeffizienten führte, wurde für die weiteren Messungen übernommen. Mit den folgenden Messparametereinstellungen wurde die Chlorophyll-Fluoreszenz der Testansätze in den Mikrotiterplatten gemessen: (1) Messmodus: Messung von unten, (2) Anre- 
gungswellenlänge: $435 \mathrm{~nm}$, Emissionswellenlänge: $685 \mathrm{~nm}$, (3) Bandbreite der Anregungs- und Emissionswellenlänge: $12 \mathrm{~nm}$, (4) Signalverstärker: 90, Anzahl der Blitze: 10, (5) Lag-Zeit: $0 \mu$ s und (6) Integrationszeit: $40 \mu$ s. Es erfolgte eine Einpunktmessung in der Mitte des Wells.

Auf der Grundlage der optimierten Messung der Chlorophyll-Fluoreszenz folgten weitere Untersuchungen zur Entwicklung bzw. Optimierung der miniaturisierten Methode: Auswahl der geeigneten Mikrotiterplatte und des entsprechenden Verschlusssystems, Überprüfung der initialen Zellkonzentration, Steigerung der Biomasseproduktion durch $\mathrm{CO}_{2}$-Begasung, Optimierung der Inkubationsbedingungen, Verringerung der Verdunstung aus den Mikrotiterplatten, Resuspension der Algen in den Testansätzen und Überprüfung der Beeinflussung der Testergebnisse durch das Material der Mikrotiterplatte und der Verschlussfolie.

Es wurde eine optimierte Methode zur Durchführung des Algentests in 48-Well-Mikrotiterplatten entwickelt. Die Validierung der miniaturisierten Methode erfolgte durch Vergleichsuntersuchungen mit dem herkömmlichen Verfahren nach DIN. Für die parallelen Untersuchungen wurden 20 Porenwässer und fünf Einzelsubstanzen ausgewählt. Die Porenwässer wurden aus 19 sedimenten hergestellt. Die Proben stammten aus der Donau, dem Hamburger Hafen, der Kieler Förde, dem Lohmer See, dem Main, dem Starnberger See, dem Stechlinsee, dem Rhein und aus der Weser. Bei den Einzelsubstanzen handelte es sich um Kaliumdichromat (CAS-Nr. 7778-50-9), Zinksulfat(heptahydrat) (CAS-Nr. 7446-20-0), 3,5-Dichlorphenol (CAS-Nr. 59135-5), Ammoniumchlorid (CAS-Nr. 12125-02-9) und Atrazin (CAS-Nr. 1912-24-9). Zur Überprüfung des Nährstoffgehalts wurden Gesamt-Stickstoff, Ammonium-Stickstoff, Nitrat-Stickstoff, Nitrit-Stickstoff und Gesamt-Phosphat der Porenwässer mit Küvettenschnelltests (HACH LANGE $\mathrm{GmbH}$, Düsseldorf) bestimmt. Die Konzentration von Ammonium-Stickstoff wurde auch nach $72 \mathrm{~h}$ bestimmt, da sich hohe Konzentrationen wachstumshemmend auf den Testorganismus auswirken können.

Die Algenstammkultur mit dem Testorganismus Desmodesmus subspicatus wurde gemäß der Norm hergestellt und gelagert. Die Algenvorkultur für die Anzucht des Inokulums wurde drei Tage vor Beginn des Algentests in entsprechender Konzentration angesetzt und inkubiert. Somit wurde sichergestellt, dass sich die für den Test verwendeten Algen in der exponentiellen Wachstumsphase befanden. Aus der Algenvorkultur wurde das Inokulum mit einer Zelldichte von $5 \times 10^{4}$ Zellen $/ \mathrm{ml}$ hergestellt. In der Norm wird hier eine Zelldichte von $10^{5}$ Algenzellen $/ \mathrm{ml}$ vorgeschlagen, vorangegangene Untersuchungen zeigten jedoch, dass mit der Verringerung der Zelldichte eine Steigerung der Biomasseproduktion erreicht werden konnte. Da die Norm eine minimale Steigerung der Biomasseproduktion um den Faktor 30 fordert, wurde - nach der Ver- dünnung des Inokulums mit den definierten Anteilen aus Testgut, Verdünnungswasser und ISO-Nährmedium - für die Testansätze eine Algenkonzentration von $5 \times 10^{3} \mathrm{Zel}-$ len $/ \mathrm{ml}$ verwendet.

Aus den Porenwässern und den Stammlösungen der Einzelsubstanzen wurden die Testansätze hergestellt. Ein Testansatz bestand aus einem Kontrollansatz, einer Positivreferenz (Kaliumdichromat, nur bei natürlichem Testgut) und einer Verdünnungsreihe. Mit der Positivreferenz wurde ermittelt, ob die Algen in einem bestimmten Sensitivitätsbereich lagen bzw. ob es einen Hinweis auf Störungen im Testsystem gab. Die Verdünnungsstufen wurden in einer geometrischen Abfolge mit dem Verdünnungsfaktor 2 hergestellt.

Aus Gründen der Praktikabilität und basierend auf vorangegangenen Untersuchungen mit 24-Well- und 96-WellMikrotiterplatten wurden 48-Well-Mikrotiterplatten aus Polystyrol der Firma Greiner Bio-One GmbH (Frickenhausen) verwendet. Im Gegensatzzur ebenfalls untersuchten 96-WellMikrotiterplatte aus Glas (Hellma GmbH \& Co. KG, Müllheim) sind die 48-Well-Mikrotiterplatten steril verpackte Einwegartikel. Die Erstellung der Testansätze erfolgte in separaten Gefäßen, von diesen wurden die Testkonzentrationen auf die jeweiligen Replikate verteilt. Das Volumen der Testansätze in den Replikaten betrug bei der herkömmlichen Durchführung in Reagenzgläsern $4 \mathrm{ml}$ und bei der Durchführung in Mikrotiterplatten $1 \mathrm{ml}$. Bei der miniaturisierten Durchführung wurden acht Replikate für die Kontrollen und fünf Replikate für Testansätze und Positivreferenz eingesetzt, bei der DIN-Methode sechs Replikate für die Kontrollen und drei Replikate für Testansätze und Positivreferenz. Die Befüllung der 48-Well-Mikrotiterplatte erfolgte nach einem randomisierten Schema.

Verschlossen wurden die Reagenzgläser mit KapsenbergKappen und die 48-Well-Mikrotiterplatten mit permeablen BreatheEasy ${ }^{\mathrm{TM}}$-Folien (Diversified Biotech, Boston). Die Verwendung dieser Folien ist das Resultat aus den Untersuchungen zu einem geeigneten Verschlusssystem für die 48-Well-Mikrotiterplatten. Überprüft wurde auch der mitgelieferte Deckel der 48-Well-Mikrotiterplatten in Verbindung mit „Parafilm M“ (Laboratory Film, American National Can TM, Chicago, Il). Diese Art der Abdeckung wird u. a. auch von Eisenträger et al. (2003) beschrieben. Auch Paixão et al. (2008) beschreiben die Verwendung des Deckels (mit einem Kondensationsring) als Verschlusssystem für die Mikrotiterplatte. Des Weiteren wurde die gasundurchlässige EASYseal ${ }^{\mathrm{TM}}$-Folie der Firma Greiner Bio-One GmbH (Frickenhausen) auf Eignung untersucht.

Da sich der mit Parafilm verschlossene Deckel aufgrund von Kondensationströpfchen und möglichen Kreuzkontaminationen als ungeeignet erwies und aufgrund geringerer Unterschiede der am Rand befindlichen Wells wurde der Verschluss mit Folien favorisiert. Ein Vergleich der mit den jeweiligen Verschlussfolien erreichten Biomasse- 
produktion zeigte keine eindeutigen Unterschiede, sodass die permeable BreatheEasy ${ }^{\mathrm{TM}}$-Folie eingesetzt wurde, die ebenso wie der Verschluss der Reagenzgläser mit Kapsenberg-Kappen einen Gasaustausch mit der Atmosphäre zulassen soll.

Die Exposition der Testansätze in den Reagenzgläsern erfolgte in einem temperierbaren Lichtdrehschrank (Lichtthermostatschrank mit Drehgestell, Fa. Rubarth Apparate $\mathrm{GmbH}$, Laatzen), die Exposition der 48-WellMikrotiterplatten in einem temperierbaren Klimaschrank (Fa. Aqualytik GmbH \& Co., Dortmund). Vorherige Untersuchungen zeigten keine Auswirkungen einer $\mathrm{CO}_{2}$-Begasung auf die Biomasseproduktion. Demnach wurde kein zusätzliches $\mathrm{CO}_{2}$ in den Klimaschrank eingeleitet. Um die Lichtintensität im Klimaschrank zu überprüfen, wurde die Grundfläche in 12 Rasterzellen unterteilt und die Lichtintensität gemessen. Mit Reflektoren und Spiegelfolie wurde eine weitgehend homogene Beleuchtung erzeugt. Im Mittel lag die Lichtintensität bei $158 \mu \mathrm{E} / \mathrm{m}^{2} \mathrm{~s}^{-1}$. Die Lichtintensität lag um ca. $30 \%$ höher als in der DIN 38412 Teil 33 gefordert. Gemäß der Norm darf die Standardabweichung von Temperatur und Lichtintensität zwischen den einzelnen Testansätzen nicht mehr als $5 \%$ betragen, mit $3 \%$ wurde dieses Kriterium erfüllt. Eine Überprüfung der Temperaturkonstanz erfolgte mit vier identisch befüllten 48-Well-Mikrotiterplatten. Nach der Exposition zeigte das Thermometer eine Schwankung von $\pm 1{ }^{\circ} \mathrm{C}$ (bei $22^{\circ} \mathrm{C}$ ). Durch eine thermische Entkopplung der Stellplätze der Mikrotiterplatten und dem Schüttelgerät wurde eine Kondensation an der Verschlussfolie durch die Abwärme des Schüttelmotors verhindert. Zur Verringerung der Verdunstung aus den Wells wurde in den Klimaschrank ein Was- serbecken mit 101 Wasser gestellt. So wurde der durchschnittliche Gewichtsverlust pro Well nach $72 \mathrm{~h}$ von 8 auf $5 \%$ gesenkt.

Die Chlorophyll-Fluoreszenz der Testansätze wurde, ebenso wie der $\mathrm{pH}$-Wert, vor der Exposition und $72 \mathrm{~h}$ nach Teststart gemessen. Die Messung erfolgte mit Fluoreszenzfotometern: bei den Mikrotiterplatten mit dem TECAN Safire und bei den Reagenzgläsern in einer Glasküvette mit definierter Schichtdicke mit dem Hitachi 2000 der Firma Hitachi Ltd. (Japan). Die Anregungs- und Emissionswellenlänge betrug 435 bzw. $685 \mathrm{~nm}$.

Um eine homogene Verteilung der Algenzellen zu gewährleisten, wurden die Reagenzgläser und die 48-Well-Mikrotiterplatten vor der Chlorophyllmessung aufgeschüttelt. Eisenträger et al. (2003) verwenden dazu bei der miniaturisierten Methode den internen Schüttler des FluoreszenzSpektrofotometers während die Homogenisierung bei Geis et al. (2000) durch mehrmaliges Aufziehen mit einer Pipette erfolgt. Die jeweils zu messenden Reagenzgläser und die Mikrotiterplatten (mit Verschlussfolie) wurden dazu ca. $1 \mathrm{~s}$ mittels eines Kreisschüttlers (Modell „Reax Top“, Heidolph Instruments $\mathrm{GmbH} \&$ Co.KG, Schwabach) geschüttelt. Bei den Mikrotiterplatten gelangen die Testansätze zwar in Kontakt mit der Verschlussfolie, da aber das Aufschütteln nur unmittelbar vor der Endmessung durchgeführt wird, waren keine negativen Effekte zu erwarten. In den vorangegangenen Untersuchungen zeigte sich beim Aufschütteln der Algen mit dem eingebauten Schüttler ein größerer Variationskoeffizient (ca. 5\%) als beim Aufschütteln mit dem Kreisschüttler „Reax Top“ (ca. 1\%). Einen Überblick über die Durchführungen der herkömmlichen und der miniaturisierten Methode gibt Tabelle 1.
Tabelle 1 Überblick über die Durchführung der herkömmlichen Methode nach DIN und die miniaturisierte Methodendurchführung in 48-WellMikrotiterplatten

\begin{tabular}{lll}
\hline $\begin{array}{l}\text { Übersicht über die angewandten } \\
\text { Methoden und deren Unterscheide }\end{array}$ & DIN-Methode & Miniaturisierte Methode \\
\hline Testgefäß & Reagenzglas & \\
Material des Testgefäßes & Glas & 48 -Well-Mikrotiterplatte \\
Testvolumen & $4 \mathrm{ml}$ & Polystyrol \\
Verschlusssystem & Kapsenberg-Kappen & $1 \mathrm{ml}$ \\
Anzahl der Replikate & Kontrollansatz 6 & Permeable Klebefolien \\
& Testansatz: jeweils 3 & Kontrollansatz 8 \\
& Positivreferenz: 3 & Testansatz: jeweils 5 \\
Inkubationsraum & Lichtthermostatschrank mit & Positivreferenz: 5 \\
& Drehgestell, Fa. Rubarth Apparate & Temperierbarer Klimaschrank, Aqualytik GmbH \& Co., \\
& GmbH, Laatzen & Dortmund \\
Beleuchtungsmittel & Osram LF25 & Osram Basic T8, LF 640 \\
Raumbeleuchtungsstärke & ca. $120 \mu$ E $/ \mathrm{m}^{2} \mathrm{~s}^{-1}$ & ca 160 $\mu$ E/m ${ }^{2} \mathrm{~s}^{-1}$ \\
Temperatur & $22 \pm 1{ }^{\circ} \mathrm{C}$ & $22 \pm 1{ }^{\circ} \mathrm{C}$ \\
Besonderheiten & - & Erhöhung der Luftfeuchte im \\
& & Inkubationsraum \\
Fluoreszenz-Spektrofotometer & Hitachi 2000, Hitachi Ltd., Japan; & TECAN Safire, TECAN \\
& Messung in Glasküvette mit defi- & GmbH, Crailsheim; direkte \\
& nierte Schichtdicke & Messung in Mikrotiterplatte \\
\hline
\end{tabular}


Ein Algentest wird als gültig angesehen, wenn drei Bedingungen eingehalten werden: (1) die Chlorophyll-Fluoreszenz im Kontrollansatz soll sich nach $72 \mathrm{~h}$ mindestens um den Faktor 30 erhöht haben (DIN 38412 Teil 33 1991), (2) bei den Kontrollansätzen darf die Abweichung des höchsten und des niedrigsten Wertes von ihrem Mittelwert maximal 7,5\% betragen (AQS-Merkblatt P-9/3 1998) und (3) $0,5 \mathrm{mg} / 1$ Kaliumdichromat sollen 30 bis $80 \%$ Hemmung verursachen (AQS-Merkblatt P-9/3 1998).

Aus den Ergebnissen der Algenteste mit den Umweltproben wurden die Hemmwirkungen bzw. die Fördereffekte (negatives Vorzeichen) auf die Biomasseproduktion berechnet und die pT-Werte abgeleitet. Für die Einzelsubstanzen wurden anhand der ermittelten Hemmwirkungen bzw. Fördereffekte (negatives Vorzeichen) Konzentrationswirkungskurven erstellt und EC50-Werte berechnet. Dazu wurde eine logistische Modellfunktion verwendet (OECD 1984). Die logistische Kurve des 3-parametrischen Modells Gl. 1 wurde über die Parameter $x_{0}$ und $b$ an die Ergebnisse angepasst.

$$
y=\frac{c}{1+\left(\frac{x}{x_{0}}\right)^{b}}
$$

wobei

$y=$ Hemmwirkung auf die Biomasseproduktion in \%,

$x=$ eingesetzte Wirkstoffkonzentration,

$x_{0}=$ errechnete Konzentration, bei der eine Hemmwirkung der Biomasseproduktion durch die Einzelsubstanz vorliegt (EC50),

$c=$ erwartete Hemmwirkung für $\mathrm{x}=0$,

$b=$ Steigungsparameter der Kurve.

Mit dem Mann-Whitney-U-Test, einem parameterfreien statistischen Test für unabhängige Stichproben (Janssen und Laatz 2003) wurde überprüft, ob zwischen zwei Hemmwirkungen, die sowohl mit der Durchführung des Algentests im Reagenzglas als auch in der 48-Well-Mikrotiterplatte ermittelt wurden, ein signifikanter Unterschied besteht (Bahrenberg et al. 1992; Hoffmann 2000). Der U-Test setzt annähernd gleiche Stichprobenverteilungsformen voraus (Lozán und Kausch 2007). Die zwei getesteten Stichproben Hemmwerte aus dem Grünalgentest in Reagenzgläsern und in Mikrotiterplatten wiesen allerdings in der Verteilungsform z. T. Unterschiede auf. Mittels der Software SPSS 15.0 (SPSS Inc., Illinois) können jedoch selbst bei Abweichungen von den Annahmebedingungen des Testverfahrens akkurate Ergebnisse erzielt werden. Dies erfolgt unter Anwendung einer Methodenerweiterung, die als „exakte Testmethode“ bezeichnet wird (Mehta et al. 1995). Mit dieser wurden $p$-Werte berechnet. Diese kennzeichnen die Irrtumswahrscheinlichkeit, dass die Nullhypothese H0 abgelehnt wird, obwohl sie richtig ist ( $\alpha$-Fehler). Die $p$-Werte werden mit dem Signifikanzniveau $\alpha=0,05$ verglichen: ist $p \leq \alpha$, so unterscheiden sich die Hemmwirkungen im Reagenzglas und in der 48-Well-Mikrotiterplatte.

\section{Ergebnisse}

Zur Überprüfung des Nährstoffgehaltes wurde das Testgut analysiert. Folgende Konzentrationsbereiche wurden bei den natürlichen Porenwässern gemessen: Gesamt-Stickstoff $\left(\mathrm{TN}_{\mathrm{b}}\right)$ im Bereich von 0,69 bis 59,2 mg/l, AmmoniumStickstoff $\left(\mathrm{NH}_{4}-\mathrm{N}\right)$ im Bereich von 1,22 bis $53,7 \mathrm{mg} / \mathrm{l}$, Nitrat-Stickstoff $\left(\mathrm{NO}_{3}-\mathrm{N}\right)$ im Bereich von 0,11 bis $13,2 \mathrm{mg} / \mathrm{l}$, Nitrit-Stickstoff $\left(\mathrm{NO}_{2}-\mathrm{N}\right)$ im Bereich von 0,02 bis $0,19 \mathrm{mg} / \mathrm{l}$ und Phosphat-P gesamt $\left(\mathrm{PO}_{4}-\mathrm{P}\right)$ im Bereich von 0,02 bis $4,33 \mathrm{mg} / \mathrm{l}$.

Die Testgüter wurden zeitgleich im herkömmlichen Verfahren mit einem Testvolumen von $4 \mathrm{ml}$ im Reagenzglas und mit dem Mikrotiterplatten-Verfahren mit einem Testvolumen von $1 \mathrm{ml}$ in der Mikrotiterplatte (48-Well) getestet, da sich die Eigenschaften von Porenwässern mit der Zeit verändern können. Als Testgut wurden 20 Porenwässer (jeweils einmal untersucht) und fünf Einzelsubstanzen (jeweils doppelt untersucht) verwendet. Ausgewertet wurden die ermittelten Hemmwirkungen, die Differenz der Mittelwerte sowie der $p$-Wert. Des Weiteren wurden die pT-Werte für Porenwässer und die EC50-Werte für die Einzelsubstanzen berechnet.

Bei beiden Methodendurchführungen sind Fördereffekte aufgetreten. Diese waren vor allem bei den Umweltproben zu beobachten. Die Standardabweichungen der Replikate lagen für die Mikrotiterplatten im Durchschnitt bei 4,9\% und für die Methode mit Reagenzgläsern bei 5,1\% (Tabelle 2).

Bei den Hemmwirkungen lagen die Standardabweichungen der Replikate der Einzelsubstanzen bei der Mikrotiterplatte bei durchschnittlich 3,5\% und bei der herkömmlichen Methode im Reagenzglas bei 2,6\% (Tabelle 3).

Zum Vergleich der parallel durchgeführten Algentests in Reagenzgläsern und in 48-Well-Mikrotiterplatten wurde die Differenz des Mittelwerts der Hemmwirkungen einer Verdünnungsstufe berechnet. Die geringste Differenz des Mittelwertes der Hemmwirkungen in der Mikrotiterplatte im Vergleich zur DIN-Methode beträgt 0 Prozentpunkte, die größte Differenz beträgt 100 Prozentpunkte (Tabelle 4, 5). Die durchschnittliche Differenz zwischen den zwei Methoden lag bei 21 Prozentpunkten.

Bei der Überprüfung der Nullhypothese H0 mit dem Mann-Whitney-U-Test wird deutlich, dass sich die mit beiden Methoden erzielten Hemmwirkungen bzw. Fördereffekte für eine Verdünnungsstufe z. T. statistisch signifikant unterscheiden: Bei den Algentests mit Umweltproben wurden in 100 von 147 Fällen signifikante Unterschiede zwischen beiden Methoden beobachtet (siehe Tabelle 4), bei den Algentests mit Einzelsubstanzen in 55 von 80 Fällen (siehe Tabelle 5). 
Tabelle 2 Ergebnisse der Algentests mit Umweltproben nach der miniaturisierten Methodendurchführung in 48-Well-Mikrotiterplatten (MTP) und nach der herkömmlichen Methode nach DIN (DIN). Dargestellt sind die Hemmwirkungen (H\%). Die Fördereffekte sind mit negativem Vorzeichen gekennzeichnet. Die Standardabweichung (StAbw.) bezieht sich auf die Replikate

Hemmwirkung der Biomasseproduktion und Standardabweichung pro Verdünnungsstufe

(MTP: miniaturisierte Methodendurchführung in der Mikrotiterplatte, DIN: Normverfahren in Reagenzgläschen)

\begin{tabular}{|c|c|c|c|c|c|c|c|c|c|c|c|c|c|c|c|}
\hline \multicolumn{2}{|c|}{$\begin{array}{l}\text { Bezeichnung der } \\
\text { Umweltprobe }\end{array}$} & \multicolumn{2}{|c|}{$\begin{array}{c}\text { Verdünnung } \\
1: 1,25\end{array}$} & \multicolumn{2}{|c|}{$\begin{array}{c}\text { Verdünnung } \\
1: 2\end{array}$} & \multicolumn{2}{|c|}{$\begin{array}{c}\text { Verdünnung } \\
1: 4\end{array}$} & \multicolumn{2}{|c|}{$\begin{array}{c}\text { Verdünnung } \\
1: 8\end{array}$} & \multicolumn{2}{|c|}{$\begin{array}{c}\text { Verdünnung } \\
1: 16\end{array}$} & \multicolumn{2}{|c|}{$\begin{array}{c}\text { Verdünnung } \\
1: 32\end{array}$} & \multicolumn{2}{|c|}{$\begin{array}{c}\text { Verdünnung } \\
\text { 1:64 }\end{array}$} \\
\hline & & МТР & DIN & МТР & DIN & МТP & DIN & МТР & DIN & MTP & DIN & МТР & DIN & MTP & DIN \\
\hline \multirow[t]{2}{*}{ Probe A } & $\mathrm{H} \%$ & -74 & -14 & -10 & -7 & -41 & -30 & -37 & -37 & -33 & -38 & -10 & -33 & -5 & -29 \\
\hline & StAbw. & 22 & 3 & 7 & 3 & 6 & 4 & 4 & 1 & 2 & 4 & 4 & 4 & 13 & 1 \\
\hline \multirow[t]{2}{*}{ Probe B } & $\mathrm{H} \%$ & 14 & -10 & -20 & -19 & -19 & -25 & -17 & -28 & -3 & -20 & 8 & -12 & 5 & -6 \\
\hline & StAbw. & 6 & 1 & 6 & 4 & 2 & 6 & 3 & 2 & 1 & 5 & 3 & 1 & 3 & 2 \\
\hline \multirow[t]{2}{*}{ Probe C } & $\mathrm{H} \%$ & -3 & 25 & -29 & 16 & -20 & -11 & -17 & -24 & -9 & -16 & 3 & -14 & 3 & -11 \\
\hline & StAbw. & 6 & 20 & 6 & 20 & 4 & 27 & 8 & 1 & 4 & 2 & 10 & 3 & 8 & 2 \\
\hline \multirow[t]{2}{*}{ Probe D } & $\mathrm{H} \%$ & -8 & -7 & -30 & -13 & -30 & -32 & -26 & -47 & -17 & -36 & -8 & -32 & -5 & -20 \\
\hline & StAbw. & 34 & 6 & 6 & 5 & 5 & 4 & 8 & 4 & 3 & 2 & 4 & 2 & 5 & 2 \\
\hline \multirow[t]{2}{*}{ Probe E } & $\mathrm{H} \%$ & 3 & -11 & -21 & -34 & -22 & -26 & -21 & -12 & -15 & -7 & 0 & -8 & 0 & -7 \\
\hline & StAbw. & 3 & 5 & 3 & 2 & 6 & 6 & 4 & 4 & 2 & 2 & 4 & 4 & 6 & 4 \\
\hline \multirow[t]{2}{*}{ Probe F } & $\mathrm{H} \%$ & 6 & -5 & 5 & -11 & -22 & -12 & -20 & -19 & -12 & -14 & -3 & -15 & -5 & -7 \\
\hline & StAbw. & 5 & 5 & 3 & 1 & 3 & 1 & 6 & 3 & 10 & 2 & 2 & 2 & 5 & 2 \\
\hline \multirow[t]{2}{*}{ Probe G } & $\mathrm{H} \%$ & 100 & 100 & 100 & 100 & 100 & 100 & 91 & 100 & 39 & 100 & -63 & 83 & 2 & 81 \\
\hline & StAbw. & 0 & 0 & 0 & 0 & 0 & 0 & 1 & 0 & 7 & 0 & 3 & 1 & 4 & 5 \\
\hline \multirow[t]{2}{*}{ Probe $\mathrm{H}$} & $\mathrm{H} \%$ & 62 & 65 & 47 & 34 & 8 & 0 & -15 & -10 & -13 & -22 & -6 & -29 & 5 & -25 \\
\hline & StAbw. & 5 & 2 & 4 & 7 & 3 & 2 & 6 & 1 & 7 & 3 & 7 & 2 & 6 & 1 \\
\hline \multirow[t]{2}{*}{ Probe I } & $\mathrm{H} \%$ & 91 & 94 & 68 & 87 & -3 & 51 & -31 & -2 & -25 & -2 & -12 & -3 & -10 & -6 \\
\hline & StAbw. & 2 & 0 & 2 & 1 & 3 & 3 & 4 & 3 & 10 & 1 & 4 & 4 & 4 & 3 \\
\hline \multirow[t]{2}{*}{ Probe J } & $\mathrm{H} \%$ & 96 & 98 & 80 & 90 & 2 & 51 & -33 & 1 & -22 & -6 & -11 & -5 & -34 & -16 \\
\hline & StAbw. & 0 & 0 & 1 & 2 & 5 & 3 & 4 & 5 & 3 & 4 & 3 & 7 & 7 & 4 \\
\hline \multirow[t]{2}{*}{ Probe K } & $\mathrm{H} \%$ & 85 & 87 & 46 & 78 & -118 & -63 & -41 & -21 & -33 & -28 & -14 & -15 & -7 & -2 \\
\hline & StAbw. & 1 & 1 & 2 & 3 & 10 & 6 & 2 & 3 & 5 & 3 & 2 & 4 & 2 & 4 \\
\hline \multirow[t]{2}{*}{ Probe L } & $\mathrm{H} \%$ & 25 & -9 & -16 & -43 & -21 & -52 & -28 & -29 & -20 & -27 & -3 & -46 & -9 & -81 \\
\hline & StAbw. & 1 & 2 & 5 & 7 & 6 & 13 & 3 & 3 & 3 & 7 & 5 & 12 & 11 & 8 \\
\hline Probe $\mathrm{M}$ & H\% & -6 & -6 & -34 & -43 & -29 & -32 & -22 & -24 & -11 & -23 & 0 & -22 & -2 & -37 \\
\hline & StAbw. & 7 & 8 & 5 & 1 & 6 & 8 & 8 & 5 & 4 & 10 & 4 & 5 & 2 & 5 \\
\hline Probe $\mathrm{N}$ & $\mathrm{H} \%$ & 47 & 30 & 36 & 2 & 17 & 10 & -14 & -11 & -11 & -27 & 4 & -22 & 6 & -38 \\
\hline & StAbw. & 2 & 3 & 6 & 2 & 6 & 8 & 1 & 3 & 5 & 1 & 4 & 0 & 1 & 12 \\
\hline Probe $\mathrm{O}$ & $\mathrm{H} \%$ & 15 & -4 & -10 & -26 & -14 & -14 & -8 & -1 & -6 & -25 & 0 & -23 & 0 & 0 \\
\hline & StAbw. & 3 & 4 & 5 & 4 & 2 & 11 & 4 & 10 & 4 & 6 & 4 & 2 & 6 & 5 \\
\hline Probe P & $\mathrm{H} \%$ & -18 & 24 & -21 & -20 & -17 & -43 & -16 & -15 & -4 & 1 & 3 & -20 & 0 & -13 \\
\hline & StAbw. & 10 & 11 & 7 & 15 & 4 & 13 & 4 & 9 & 5 & 7 & 5 & 4 & 6 & 8 \\
\hline Probe Q & $\mathrm{H} \%$ & 14 & 43 & -1 & 22 & -3 & -21 & -25 & -5 & -18 & 6 & 1 & 2 & -3 & 8 \\
\hline & StAbw. & 4 & 1 & 4 & 14 & 4 & 9 & 3 & 11 & 6 & 1 & 7 & 5 & 8 & 5 \\
\hline Probe R & $\mathrm{H} \%$ & -12 & -68 & -10 & -78 & -13 & -23 & -10 & -67 & -5 & -70 & 8 & -45 & 9 & -9 \\
\hline & StAbw. & 3 & 13 & 2 & 19 & 6 & 4 & 3 & 25 & 3 & 8 & 1 & 11 & 5 & 5 \\
\hline Probe $\mathrm{S}$ & $\mathrm{H} \%$ & 29 & 11 & 12 & -5 & -5 & 28 & -15 & 15 & -41 & -4 & -15 & -38 & -18 & -42 \\
\hline & StAbw. & 3 & 4 & 4 & 1 & 7 & 10 & 7 & 8 & 5 & 5 & 4 & 12 & 19 & 12 \\
\hline Probe $\mathrm{S}$ & $\mathrm{H} \%$ & 28 & 7 & 6 & -7 & -14 & 30 & -15 & -35 & -28 & -6 & -17 & -34 & -13 & -33 \\
\hline Wdh.) & StAbw. & 8 & 3 & 6 & 4 & 5 & 1 & 4 & 13 & 7 & 3 & 8 & 7 & 2 & 5 \\
\hline
\end{tabular}

(Erweiterung zu Verdünnung Verdünnung Verdünnung Verdünnung Verdünnung Verdünnung Verdünnung

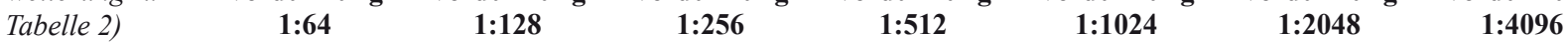

Bez. d. Umweltprobe

$\begin{array}{lllllllllllll}\text { MTP DIN } & \text { MTP } & \text { DIN } & \text { MTP } & \text { DIN } & \text { MTP } & \text { DIN } & \text { MTP } & \text { DIN } & \text { MTP } & \text { DIN } & \text { MTP } & \text { DIN }\end{array}$

\begin{tabular}{lllllllllllllllllllll}
\hline Probe $\mathrm{G}$ & $\mathrm{H} \%$ & 3 & 98 & -8 & 92 & -4 & 41 & -13 & 21 & -13 & 8 & -2 & 9 & -5 & 0
\end{tabular}

$\begin{array}{llllllllllllllll}\text { (Erw.) } & \text { StAbw. } & 3 & 0 & 3 & 3 & 2 & 2 & 4 & 11 & 2 & 3 & 7 & 11 & 7 & 1\end{array}$


Tabelle 3 Ergebnisse der Algentests mit Einzelsubstanzen nach der miniaturisierten Methodendurchführung in 48-Well-Mikrotiterplatten (MTP) und nach der herkömmlichen Methode nach DIN (DIN). Dargestellt sind die Hemmwirkungen (H\%). Die Fördereffekte sind mit negativem Vorzeichen gekennzeichnet, Wiederholungsuntersuchungen durch A und B. Die Standardabweichung (StAbw.) bezieht sich auf die Replikate

Hemmwirkung der Biomasseproduktion und Standardabweichung pro Verdünnungsstufe (MTP: miniaturisierte Methodendurchführung in der Mikrotiterplatte, DIN: Normverfahren in Reagenzgläschen)

\begin{tabular}{|c|c|c|c|c|c|c|c|c|c|c|c|c|c|c|c|c|c|}
\hline \multicolumn{2}{|c|}{ Kaliumdichromat } & \multicolumn{2}{|c|}{$2 \mathrm{mg} / \mathrm{l}$} & \multicolumn{2}{|c|}{$1 \mathrm{mg} / \mathrm{l}$} & \multicolumn{2}{|c|}{$0,7 \mathrm{mg} / \mathrm{l}$} & \multicolumn{2}{|c|}{$0,5 \mathrm{mg} / \mathrm{l}$} & \multicolumn{2}{|c|}{$0,35 \mathrm{mg} / \mathrm{l}$} & \multicolumn{2}{|c|}{$0,25 \mathrm{mg} / \mathrm{l}$} & \multicolumn{2}{|c|}{$0,175 \mathrm{mg} / \mathrm{l}$} & \multicolumn{2}{|c|}{$0,125 \mathrm{mg} / \mathrm{l}$} \\
\hline & & MTP & DIN & МTP & DIN & МTP & DIN & MTP & DIN & МTP & DIN & МTP & DIN & МTP & DIN & МТP & DIN \\
\hline $\mathrm{K}_{2} \mathrm{Cr}_{2} \mathrm{O}_{7} \quad \mathrm{~A}$ & $\mathrm{H} \%$ & 93 & 96 & 81 & 84 & 71 & 69 & 50 & 59 & 12 & 32 & 7 & 17 & -2 & 5 & -1 & 1 \\
\hline & StAbw. & 0 & 0 & 1 & 0 & 2 & 1 & 2 & 1 & 3 & 1 & 6 & 3 & 7 & 3 & 8 & 2 \\
\hline $\mathrm{K}_{2} \mathrm{Cr}_{2} \mathrm{O}_{7} \quad \mathrm{~B}$ & $\mathrm{H} \%$ & 93 & 96 & 83 & 86 & 71 & 72 & 51 & 56 & 15 & 20 & 15 & 1 & 7 & -5 & 6 & -5 \\
\hline & StAbw. & 1 & 0 & 0 & 2 & 1 & 2 & 2 & 0 & 3 & 2 & 4 & 5 & 9 & 1 & 10 & 6 \\
\hline \multirow{2}{*}{\multicolumn{2}{|c|}{$\begin{array}{l}\text { Zinksulfat- } \\
\text { Heptahydrat }\end{array}$}} & \multicolumn{2}{|c|}{$300 \mathrm{mg} / \mathrm{l}$} & \multicolumn{2}{|c|}{$100 \mathrm{mg} / \mathrm{l}$} & \multicolumn{2}{|c|}{$33,3 \mathrm{mg} / \mathrm{l}$} & \multicolumn{2}{|c|}{$11,1 \mathrm{mg} / \mathrm{l}$} & \multicolumn{2}{|c|}{$3,7 \mathrm{mg} / \mathrm{l}$} & \multicolumn{2}{|c|}{$1,23 \mathrm{mg} / \mathrm{l}$} & \multicolumn{2}{|c|}{$0,41 \mathrm{mg} / \mathrm{l}$} & \multicolumn{2}{|c|}{$0,14 \mathrm{mg} / \mathrm{l}$} \\
\hline & & MTP & DIN & MTP & DIN & MTP & DIN & МTP & DIN & MTP & DIN & МТP & DIN & MTP & DIN & MTP & DIN \\
\hline $\begin{array}{c}\mathrm{ZnSO}_{4} \times 7 \mathrm{H}_{2} \mathrm{O} \\
\mathrm{A}\end{array}$ & $\mathrm{H} \%$ & 99 & 99 & 99 & 99 & 99 & 99 & 99 & 99 & 99 & 99 & 81 & 71 & 62 & 45 & 18 & -8 \\
\hline & StAbw. & 0 & 0 & 0 & 0 & 0 & 0 & 0 & 0 & 0 & 0 & 1 & 0 & 3 & 4 & 2 & 5 \\
\hline $\mathrm{ZnSO}_{4} \times 7 \mathrm{H}_{2} \mathrm{O}$ & $\mathrm{H} \%$ & 99 & 98 & 99 & 100 & 98 & 95 & 82 & 79 & 49 & 34 & 25 & -5 & -3 & -7 & -7 & -1 \\
\hline B & StAbw. & 0 & 0 & 0 & 0 & 0 & 0 & 1 & 1 & 3 & 4 & 3 & 0 & 5 & 2 & 6 & 5 \\
\hline \multirow{2}{*}{\multicolumn{2}{|c|}{ Dichlorphenol }} & \multicolumn{2}{|c|}{$18 \mathrm{mg} / \mathrm{l}$} & \multicolumn{2}{|c|}{$13,5 \mathrm{mg} / \mathrm{l}$} & \multicolumn{2}{|c|}{$9 \mathrm{mg} / \mathrm{l}$} & \multicolumn{2}{|c|}{$6,75 \mathrm{mg} / \mathrm{l}$} & \multicolumn{2}{|c|}{$4,5 \mathrm{mg} / \mathrm{l}$} & \multicolumn{2}{|c|}{$3,38 \mathrm{mg} / \mathrm{l}$} & 2,25 & ng/l & 1,69 & ng/l \\
\hline & & МTP & DIN & МTP & DIN & MTP & DIN & MTP & DIN & MTP & DIN & MTP & DIN & МTP & DIN & MTP & DIN \\
\hline $3,5-\mathrm{DCP} \quad \mathrm{A}$ & $\mathrm{H} \%$ & 100 & 95 & 99 & 75 & 93 & 40 & 63 & 15 & -4 & 0 & -24 & 1 & -19 & -1 & -33 & -6 \\
\hline & StAbw. & 0 & 0 & 1 & 0 & 0 & 1 & 2 & 1 & 5 & 2 & 5 & 5 & 6 & 3 & 4 & 1 \\
\hline $3,5-\mathrm{DCP} \quad \mathrm{B}$ & $\mathrm{H} \%$ & 94 & 99 & 54 & 96 & -24 & 84 & -26 & 38 & -25 & -4 & -30 & -16 & -31 & -12 & -26 & -4 \\
\hline & StAbw. & 4 & 0 & 21 & 0 & 9 & 0 & 3 & 1 & 2 & 2 & 2 & 2 & 2 & 13 & 3 & 12 \\
\hline Ammoniumcl & hlorid & $400 r$ & ng/l & 250 & $\mathrm{ng} / \mathrm{l}$ & $125 r$ & $\mathrm{ng} / \mathbf{l}$ & 62,5 & ng/l & 31,3 & ng/l & 15,6 & mg/l & $7,8 \mathrm{r}$ & ng/l & 3,9 r & Ig/l \\
\hline & & MTP & DIN & МTP & DIN & MTP & DIN & МTP & DIN & MTP & DIN & MTP & DIN & MTP & DIN & MTP & DIN \\
\hline $\mathrm{NH}_{4} \mathrm{Cl} \quad \mathrm{A}$ & $\mathrm{H} \%$ & 89 & 97 & 70 & 97 & 53 & 91 & 40 & 77 & 22 & 40 & 10 & 16 & 0 & 3 & -4 & 3 \\
\hline & StAbw. & 1 & 0 & 3 & 1 & 6 & 1 & 4 & 0 & 3 & 2 & 4 & 2 & 6 & 2 & 9 & 3 \\
\hline $\mathrm{NH}_{4} \mathrm{Cl} \quad \mathrm{B}$ & $\mathrm{H} \%$ & 89 & 95 & 72 & 92 & 60 & 84 & 42 & 78 & 20 & 56 & 21 & 43 & 2 & 9 & 3 & -51 \\
\hline & StAbw. & 1 & 0 & 2 & 0 & 2 & 2 & 3 & 2 & 3 & 5 & 3 & 3 & 9 & 11 & 6 & 15 \\
\hline Atrazin & & $0,4 n$ & $\mathrm{gg} / \mathrm{l}$ & 0,2 r & $\mathrm{g} / \mathrm{l}$ & $0,1 \mathrm{n}$ & $\mathrm{ng} / \mathbf{l}$ & 0,05 & ng/l & 0,025 & $\mathrm{mg} / \mathrm{l}$ & 0,012 & $\mathrm{mg} / \mathrm{l}$ & 0,006 & $\mathrm{mg} / \mathrm{l}$ & 0,003 & $\mathrm{mg} / \mathrm{l}$ \\
\hline & & МTP & DIN & МTP & DIN & MTP & DIN & МTP & DIN & МTP & DIN & МTP & DIN & МTP & DIN & МTP & DIN \\
\hline Atrazin A & $\mathrm{H} \%$ & 95 & 97 & 88 & 98 & 59 & 95 & -14 & 73 & -62 & 22 & -56 & 1 & -34 & -10 & -20 & -13 \\
\hline & StAbw. & 0 & 1 & 1 & 0 & 3 & 0 & 5 & 2 & 5 & 6 & 2 & 3 & 8 & 7 & 4 & 7 \\
\hline Atrazin B & $\mathrm{H} \%$ & 94 & 97 & 87 & 97 & 54 & 92 & -31 & 67 & -76 & 6 & -60 & -14 & -35 & -23 & -22 & -26 \\
\hline & StAbw. & 0 & 0 & 1 & 0 & 4 & 1 & 4 & 2 & 4 & 6 & 5 & 12 & 8 & 13 & 6 & 8 \\
\hline
\end{tabular}

Tabelle $6 \mathrm{~b}$ enthält die Häufigkeiten der ermittelten pTWerte aus den parallel durchgeführten Algentestmethoden. In zehn von 20 Fällen war der pT-Wert mit beiden Methodendurchführungen identisch, in acht von 20 Fällen unterschieden sich die Ergebnisse um eine Stufe, in einem Fall um zwei Stufen und in einem Fall um sechs Stufen (Tabelle $6 a)$.

Der Vergleich der EC50-Werte der Einzelsubstanzen zeigte für Kaliumdichromat, Zinksulfat und Dichlorphenol ge- ringe Abweichungen der gemittelten EC50-Werte max. um den Faktor 2. Für Ammoniumchlorid wurden mit dem Faktor 4 die größten Abweichungen festgestellt (Tabelle 6c).

Das erste Gültigkeitskriterium, die Erhöhung der Biomasse im Kontrollansatz nach $72 \mathrm{~h}$ mindestens um den Faktor 30, wurde bei der herkömmlichen Durchführung ausnahmslos erfüllt. Beim Algentest in der Mikrotiterplatte wurde es im Gegensatz dazu in 29 von 31 Fällen nicht erfüllt. 
Tabelle 4 Unterschiede der Algentests mit Umweltproben nach der miniaturisierten Methodendurchführung in 48-Well-Mikrotiterplatten (MTP) und nach der herkömmlichen Methode nach DIN (DIN). Dargestellt sind die Differenzen der Hemmwirkungen (Diff. \%).

\begin{tabular}{|c|c|c|c|c|c|c|c|c|}
\hline \multicolumn{9}{|c|}{ Differenz der gemittelten Hemmwirkungen (\%) } \\
\hline \multicolumn{2}{|c|}{$\begin{array}{l}\text { Bezeichnung } \\
\text { der Umweltprobe }\end{array}$} & $\begin{array}{c}\text { Verdünnung } \\
1: 1,25\end{array}$ & $\begin{array}{c}\text { Verdünnung } \\
1: 2\end{array}$ & $\begin{array}{c}\text { Verdünnung } \\
1: 4\end{array}$ & $\begin{array}{c}\text { Verdünnung } \\
1: 8\end{array}$ & $\begin{array}{c}\text { Verdünnung } \\
1: 16\end{array}$ & $\begin{array}{c}\text { Verdünnung } \\
1: 32\end{array}$ & $\begin{array}{c}\text { Verdünnung } \\
1: 64\end{array}$ \\
\hline Probe A & Diff. & $60^{*}$ & 3 & $11^{*}$ & 0 & 5 & $23 *$ & 14 \\
\hline Probe B & Diff. & $24 *$ & 1 & 6 & $11^{*}$ & $17 *$ & $20 *$ & $11^{*}$ \\
\hline Probe $\mathrm{C}$ & Diff. & $28^{*}$ & $45^{*}$ & 9 & 7 & $7^{*}$ & $17^{*}$ & $14^{*}$ \\
\hline Probe D & Diff. & 1 & $17^{*}$ & 2 & $21^{*}$ & $19^{*}$ & $24^{*}$ & $15^{*}$ \\
\hline Probe E & Diff. & $14^{*}$ & $13^{*}$ & 4 & 9 & $8^{*}$ & 8 & 7 \\
\hline Probe F & Diff. & $11^{*}$ & $16^{*}$ & 10 & 1 & $2^{*}$ & 12 & $2 *$ \\
\hline Probe $\mathrm{G}$ & Diff. & $0^{*}$ & $0^{*}$ & $0^{*}$ & $9 *$ & $61 *$ & $146^{*}$ & $79 *$ \\
\hline Probe $\mathrm{H}$ & Diff. & 3 & $13^{*}$ & $8^{*}$ & 5 & 9 & $23 *$ & $30^{*}$ \\
\hline Probe I & Diff. & $3^{*}$ & $19^{*}$ & $54 *$ & $29 *$ & $23^{*}$ & 9 & 4 \\
\hline Probe J & Diff. & $2^{*}$ & $10^{*}$ & $49^{*}$ & $34 *$ & $16^{*}$ & 6 & $18^{*}$ \\
\hline Probe K & Diff. & $2^{*}$ & $32 *$ & $55^{*}$ & $20 *$ & 5 & 1 & 5 \\
\hline Probe L & Diff. & $34 *$ & $27 *$ & $31^{*}$ & 1 & 7 & $43^{*}$ & $72 *$ \\
\hline Probe M & Diff. & 0 & $9 *$ & 3 & 2 & 12 & $22 *$ & $35^{*}$ \\
\hline Probe N & Diff. & $17^{*}$ & $34^{*}$ & 7 & 3 & $16^{*}$ & $26^{*}$ & $44^{*}$ \\
\hline Probe $\mathrm{O}$ & Diff. & $19^{*}$ & $16^{*}$ & 0 & 7 & $19^{*}$ & $23^{*}$ & 0 \\
\hline Probe $\mathrm{P}$ & Diff. & $42^{*}$ & 1 & $26^{*}$ & 1 & 5 & $23^{*}$ & 13 \\
\hline Probe Q & Diff. & $29^{*}$ & $23^{*}$ & $18^{*}$ & $20 *$ & $24 *$ & 1 & 11 \\
\hline Probe R & Diff. & $56^{*}$ & $68^{*}$ & 10 & $57^{*}$ & $65^{*}$ & $53 *$ & $18^{*}$ \\
\hline Probe $\mathrm{S}$ & Diff. & $18^{*}$ & $17 *$ & $33^{*}$ & $30^{*}$ & $37 *$ & $23 *$ & 24 \\
\hline Probe S (Wdh.) & Diff. & $21^{*}$ & $13 *$ & $44 *$ & $50^{*}$ & $22 *$ & $17 *$ & $20 *$ \\
\hline \multicolumn{2}{|c|}{$\begin{array}{c}\text { (Erweiterung zu Tabelle 4) } \\
\text { Bez. d. Umweltprobe }\end{array}$} & $\begin{array}{c}\text { Verdünnung } \\
1: 64\end{array}$ & $\begin{array}{l}\text { Verdünnung } \\
1: 128\end{array}$ & $\begin{array}{c}\text { Verdünnung } \\
1: 256\end{array}$ & $\begin{array}{c}\text { Verdünnung } \\
1: 512\end{array}$ & $\begin{array}{c}\text { Verdünnung } \\
1: 1024\end{array}$ & $\begin{array}{l}\text { Verdünnung } \\
1: 2048\end{array}$ & $\begin{array}{c}\text { Verdünnung } \\
1: 4096\end{array}$ \\
\hline $\begin{array}{l}\text { Probe G } \\
\text { (Erw.) }\end{array}$ & Diff. & $95^{*}$ & $100^{*}$ & $45^{*}$ & $34 *$ & $21^{*}$ & 11 & 5 \\
\hline
\end{tabular}

*: Signifikanter Unterschied zwischen den mit beiden Methoden erzielten Ergebnissen (Mann-Whitney-U-Test, $\mathrm{p} \leq 0,05$ )

Das zweite Gültigkeitskriterium, die Abweichung der Kontrollreplikate um maximal 7,5\% voneinander (AQSMerkblatt P-9/3), wurde bei der Durchführung nach DIN in sieben von 31 Fällen nicht erfüllt, beim miniaturisiertem Algentest in zwei von 31 Fällen.

Das dritte Gültigkeitskriterium, die Hemmung durch die Positivreferenz um 30 bis $80 \%$ (AQS-Merkblatt P-9/3), wurde bei beiden Testdurchführungen jeweils in einem von 21 Fällen nicht erfüllt.

\section{Diskussion}

Die z. T. festgestellten Differenzen zwischen den Hemmwirkungen des Algentests nach DIN und der miniaturisierten Durchführung in Mikrotiterplatten sind wahrscheinlich auf die unterschiedlichen Bedingungen während der Inkubation zurückzuführen. Hierbei unterschieden sich die Methoden z.B. in der Reaktionskammergröße, dem Verhältnis von Gasraum zum Volumen des Testansatzes innerhalb der Reaktionskammer und dem Gasaustausch mit der Umgebung.
Der Vergleich der pT-Werte für die parallel durchgeführten Algentests zeigt für einen Teil der untersuchten Proben eine sehr gute Übereinstimmung (Tabelle 6). Ein direkter Vergleich der aus den Hemmwirkungen errechneten EC50-Werte der parallel durchgeführten Algentests zeigt mit Ausnahme von Ammonium für fast alle der geprüften Einzelstoffe eine gute Übereinstimmung (siehe Tabelle 6c). Der Unterschied der EC50-Werte bei Ammonium wurde vermutlich durch die unterschiedliche Entwicklung des pH-Wertes im Testverlauf verursacht. Ammonium kann besonders in natürlichen Testgütern als Störfaktor auftreten, da es in Abhängigkeit vom $\mathrm{pH}-$ Wert zu Ammoniak dissoziiert (Wahrendorf et al. 2005).

Bei beiden Methodendurchführungen traten bei den untersuchten Umweltproben häufig Fördereffekte auf.

Nur ca. ein Drittel der Hemmwirkungen (73 von 227) unterschieden sich bei einem Signifikanzniveau von $\alpha=0,05$ nicht signifikant voneinander. Eine Vergleichbarkeit, d.h. eine erfolgreiche Anpassung der miniaturisierten Durchführung des Algentests an den Algentest im herkömmlichen Verfahren, kann also durch die statistische Analyse nicht verifiziert werden. 
Tabelle 5 Unterschiede der Algentests mit Einzelsubstanzen nach der miniaturisierten Methodendurchführung in 48-Well-Mikrotiterplatten (MTP) und nach der herkömmlichen Methode nach DIN (DIN). Dargestellt sind die Differenzen der Hemmwirkungen (Diff. \%).

\begin{tabular}{|c|c|c|c|c|c|c|c|c|c|}
\hline \multicolumn{10}{|c|}{ Differenz der gemittelten Hemmwirkungen (\%) } \\
\hline \multicolumn{2}{|l|}{ Kaliumdichromat } & \multirow{2}{*}{$\frac{2 \mathrm{mg} / \mathrm{l}}{3^{*}}$} & \multirow{2}{*}{$\frac{1 \mathrm{mg} / \mathbf{l}}{3^{*}}$} & \multirow{2}{*}{$\begin{array}{r}\mathbf{0 , 7} \mathbf{~ m g} / \mathbf{l} \\
2\end{array}$} & \multirow{2}{*}{$\frac{\mathbf{0 , 5} \mathbf{~ m g} / \mathbf{l}}{9^{*}}$} & \multirow{2}{*}{$\frac{\mathbf{0 , 3 5} \mathbf{~ m g} / \mathbf{l}}{20^{*}}$} & \multirow{2}{*}{$\frac{\mathbf{0 , 2 5} \mathbf{~ m} / \mathbf{l}}{10}$} & \multirow{2}{*}{$\frac{\mathbf{0 , 1 7 5} \mathbf{~ m g} / \mathbf{l}}{7}$} & \multirow{2}{*}{$\frac{\mathbf{0 , 1 2 5} \mathrm{mg} / \mathbf{l}}{2}$} \\
\hline $\mathrm{K}_{2} \mathrm{Cr}_{2} \mathrm{O}_{7} \quad \mathrm{~A}$ & Diff. & & & & & & & & \\
\hline $\mathrm{K}_{2} \mathrm{Cr}_{2} \mathrm{O}_{7} \quad \mathrm{~B}$ & Diff. & $3 *$ & $3 *$ & 1 & $5^{*}$ & 5 & $14^{*}$ & 12 & 11 \\
\hline Zinksulfat-Heptahy & Arat & $300 \mathrm{mg} / \mathrm{l}$ & $100 \mathrm{mg} / \mathrm{l}$ & $33,3 \mathrm{mg} / \mathbf{l}$ & $11,1 \mathrm{mg} / \mathrm{l}$ & $3,7 \mathrm{mg} / \mathrm{l}$ & $1,23 \mathrm{mg} / \mathrm{l}$ & $0,41 \mathrm{mg} / \mathrm{l}$ & $0,14 \mathrm{mg} / \mathrm{l}$ \\
\hline $\mathrm{ZnSO}_{4} \times 7 \mathrm{H}_{2} \mathrm{O} \quad \mathrm{A}$ & Diff. & 0 & 0 & 0 & 0 & 0 & $10^{*}$ & $17 *$ & $26^{*}$ \\
\hline $\mathrm{ZnSO}_{4} \times 7 \mathrm{H}_{2} \mathrm{O} \quad \mathrm{B}$ & Diff. & 1 & $1^{*}$ & $3 *$ & $3^{*}$ & $15^{*}$ & $30^{*}$ & 4 & 6 \\
\hline Dichlorphenol & & $18 \mathrm{mg} / \mathrm{l}$ & $13,5 \mathrm{mg} / \mathrm{l}$ & $9 \mathrm{mg} / \mathrm{l}$ & $6,75 \mathrm{mg} / \mathrm{l}$ & $4,5 \mathrm{mg} / \mathrm{l}$ & $3,38 \mathrm{mg} / \mathrm{l}$ & $2,25 \mathrm{mg} / \mathrm{l}$ & $1,69 \mathrm{mg} / \mathrm{l}$ \\
\hline $3,5-\mathrm{DCP} \quad \mathrm{A}$ & Diff. & $5^{*}$ & $24 *$ & $53^{*}$ & $48^{*}$ & 4 & $25^{*}$ & $18^{*}$ & $27^{*}$ \\
\hline 3,5-DCP B & Diff. & 5 & $42 *$ & $108 *$ & $64^{*}$ & $21^{*}$ & $14^{*}$ & $19^{*}$ & $22 *$ \\
\hline Ammoniumchlorid & & $400 \mathrm{mg} / \mathrm{l}$ & $250 \mathrm{mg} / \mathrm{l}$ & $125 \mathrm{mg} / \mathrm{l}$ & $62,5 \mathrm{mg} / \mathrm{l}$ & $31,3 \mathrm{mg} / \mathrm{l}$ & $15,63 \mathrm{mg} / \mathrm{l}$ & $7,81 \mathrm{mg} / \mathrm{l}$ & $3,9 \mathrm{mg} / \mathrm{l}$ \\
\hline $\mathrm{NH}_{4} \mathrm{Cl} \quad \mathrm{A}$ & Diff. & $8^{*}$ & $27^{*}$ & $38^{*}$ & $37^{*}$ & $18^{*}$ & 6 & 3 & 7 \\
\hline $\mathrm{NH}_{4} \mathrm{Cl} \quad \mathrm{B}$ & Diff. & $6^{*}$ & $20^{*}$ & $24 *$ & $36^{*}$ & $36^{*}$ & $22^{*}$ & 7 & $54 *$ \\
\hline Atrazin & & $0,4 \mathrm{mg} / \mathrm{l}$ & $0,2 \mathrm{mg} / \mathrm{l}$ & $0,1 \mathrm{mg} / \mathrm{l}$ & $0,05 \mathrm{mg} / \mathrm{l}$ & $0,025 \mathrm{mg} / \mathrm{l}$ & $0,0125 \mathrm{mg} / \mathrm{l}$ & $0,0063 \mathrm{mg} / \mathrm{l}$ & $0,0031 \mathrm{mg} / \mathrm{l}$ \\
\hline Atrazin A & Diff. & $2 *$ & $10^{*}$ & $36^{*}$ & $87 *$ & $84 *$ & $57 *$ & $24 *$ & 7 \\
\hline Atrazin B & Diff. & $3 *$ & $10 *$ & $38^{*}$ & $98^{*}$ & $82 *$ & $46^{*}$ & 12 & 4 \\
\hline
\end{tabular}

*: Signifikanter Unterschied zwischen den mit beiden Methoden erzielten Ergebnissen (Mann-Whitney-U-Test, $\mathrm{p} \leq 0,05$ ). Wiederholungsuntersuchungen sind durch A und B gekennzeichnet

Tabelle 6 Ergebnisse der Algentests nach der miniaturisierten Methodendurchführung in 48-Well-Mikrotiterplatten (MTP) und nach der herkömmlichen Methode nach DIN (DIN). Wiederholungsuntersuchungen sind durch A und B gekennzeichnet. a) pT-Werte der Umweltproben; b) Häufigkeiten der Abweichungen der ermittelten pT-Werte; c) EC50-Werte der Einzelsubstanzen

a)

\begin{tabular}{|c|c|c|c|c|c|c|c|c|}
\hline \multirow{2}{*}{$\begin{array}{l}\text { Bezeichnung } \\
\text { der Umwelt- } \\
\text { probe }\end{array}$} & \multicolumn{2}{|c|}{ pT-Wert } & \multirow{2}{*}{$\begin{array}{l}\text { Differenz der pT- } \\
\text { Werte }\end{array}$} & \multirow{2}{*}{$\begin{array}{c}\text { Differenz der } \\
\text { pT-Werte }\end{array}$} & \multirow[t]{2}{*}{ Häufigkeit } & \multirow{2}{*}{$\begin{array}{l}\text { Bezeichnung der } \\
\text { Einzelsubstanz }\end{array}$} & \multicolumn{2}{|c|}{ EC50-Wert (mg/l) } \\
\hline & МТP & DIN & & & & & МТP & DIN \\
\hline Probe A & 0 & 0 & 0 & & & & & \\
\hline Probe B & 0 & 0 & 0 & 0 & 10 & Kaliumdichromat & & \\
\hline Probe $\mathrm{C}$ & 0 & 1 & 1 & & & $\mathrm{~K}_{2} \mathrm{Cr}_{2} \mathrm{O}_{7} \mathrm{~A}$ & 0,49 & 0,45 \\
\hline Probe D & 0 & 0 & 0 & & & $\mathrm{~K}_{2} \mathrm{Cr}_{2} \mathrm{O}_{7} \mathrm{~B}$ & 0,50 & 0,47 \\
\hline Probe E & 0 & 0 & 0 & 1 & 8 & & & \\
\hline Probe F & 0 & 0 & 0 & & & Zinksulfat-Heptahydrat & & \\
\hline Probe $\mathrm{G}$ & 5 & 11 & 6 & & & $\mathrm{ZnSO}_{4} \times 7 \mathrm{H}_{2} \mathrm{OA}$ & 0,33 & 0,57 \\
\hline Probe $\mathrm{H}$ & 2 & 2 & 0 & 2 & 1 & $\mathrm{ZnSO}_{4} \times 7 \mathrm{H}_{2} \mathrm{O} \mathrm{B}$ & 0,40 & 0,59 \\
\hline Probe I & 2 & 3 & 1 & & & & & \\
\hline Probe $\mathrm{J}$ & 2 & 3 & 1 & & & Dichlorphenol & & \\
\hline Probe K & 2 & 2 & 0 & 3 & 0 & 3,5-DCP A & 6,5 & 11,0 \\
\hline Probe L & 1 & 0 & 1 & & & 3,5-DCP B & $13,0^{1}$ & 7,1 \\
\hline Probe $\mathrm{M}$ & 0 & 0 & 0 & & & & & \\
\hline Probe N & 2 & 1 & 1 & 4 & 0 & Ammoniumchlorid & & \\
\hline Probe $\mathrm{O}$ & 0 & 0 & 0 & & & $\mathrm{NH}_{4} \mathrm{Cl} \mathrm{A}$ & 119 & 35,8 \\
\hline Probe $\mathrm{P}$ & 0 & 1 & 1 & & & $\mathrm{NH}_{4} \mathrm{Cl} \mathrm{B}$ & 113 & 21,1 \\
\hline Probe Q & 0 & 2 & 2 & 5 & 0 & & & \\
\hline Probe $\mathrm{R}$ & 0 & 0 & 0 & & & Atrazin & & \\
\hline Probe $\mathrm{S}$ & 1 & 0 & 1 & 6 & 1 & Atrazin A & 0,098 & 0,036 \\
\hline Probe S (Wdh.) & 1 & 0 & 1 & & & Atrazin B & $0,09^{1}$ & 0,043 \\
\hline
\end{tabular}




\section{Schlussfolgerungen und Ausblick}

In dieser Arbeit wurde eine Methode zur Durchführung des Algentests in der 48-Well-Mikrotiterplatte entwickelt. Zusammenfassend lässt sich festhalten, dass bei der hier vorgestellten Methode für den Algentest in der Mikroplatte noch weitere Optimierungsschritte durchgeführt werden sollten.

Das Gültigkeitskriterium der Biomassevervielfachung in den Kontrollen um den Faktor 30 wurde mit der hier vorgestellten Methode häufig nicht eingehalten. In weiteren Untersuchungen muss die methodische Vorgehensweise so angepasst werden, dass die geforderte Vervielfachung im Regelfall eingehalten wird. Eventuell kann hier der Einsatz von speziell angefertigten Glasmikrotiterplatten mit größeren Volumina zur Lösung beitragen. Die Verwendung von Glas als Material zur Exposition der Testansätze verringert außerdem eine negative Beeinflussung der Ergebnisse durch Absorptionseffekte an der Gefäßoberfläche.

Der Einsatz von Mikrotiterplatten ermöglicht die Automatisierung von Verfahrensabläufen, um somit die Effizienz des Systems zu steigern.

Danksagung Unser Dank richtet sich vor allem an Angela Koppers und Ilona Kirchesch, deren methodische Hinweise stets hilfreich waren sowie an alle weiteren Mitarbeiterinnen und Mitarbeiter des Referates G3 (Ökotoxikologie) der Bundesanstalt für Gewässerkunde.

\section{Literatur}

Ahlf W (1995) Biotest an Sedimenten. In: Steinberg C, Calmano W, Klapper H, Wilke RD: Handbuch Angewandte Limnologie Grundlagen - Gewässerbelastung - Restaurierung - Aquatische Ökotoxikologie - Bewertung - Gewässerschutz. Ecomed-Verlag, Landsberg/Lech, S 2-43

Ahlf W, Flemming H-C, Götz R, Hupfer M, Leuchs H, Lorch D, Petersen W, Traunspurger W (2001) Sedimente als Lebensraum. In: Calmano W Untersuchung und Bewertung von Sedimenten - Ökotoxikologische und chemische Testmethoden. SpringerVerlag, Berlin Heidelberg New York, S 7-66

AQS-Merkblatt P-9/3 (1998) Bestimmung der nicht giftigen Wirkung von Abwasser gegenüber Grünalgen (Scenedesmus-ChlorophyllFluoreszenztest) über Verdünnungsstufen (DIN 38412 - L33). Bund/Länder-Arbeitsgemeinschaft Wasser (LAWA), Berlin

Bahrenberg G, Giese E, Nipper J (1992) Statistische Methoden in der Geographie, Bd. 2, Multivariate Statistik, 2. Aufl. Teubner Verlag, Stuttgart

Bundesanstalt für Gewässerkunde, G3 Ökologie (Stand 2007) BfGMerkblatt „Ökotoxikologische Baggergutuntersuchungen“, Ökotoxikologische Untersuchung von Sedimenten, Eluaten und Porenwässern. Koblenz

Cleuvers M (2002) Aquatische Ökotoxikologie ausgewählter Arzneimittel - Algentest und akuter Daphnientest. Z Umweltchem Ökotox $14(2): 85-89$

DIN 38412 Teil 33 (1991) Testverfahren mit Wasserorganismen (Gruppe L) - Bestimmung der nicht giftigen Wirkung von Abwasser gegenüber Grünalgen (Scenedesmus-Chlorophyll-Fluoreszenztest) über Verdünnungsstufen (L33). Deutsche Einheitsverfahren zur Wasser,- Abwasser- und Schlammuntersuchung. Bd. VI K-L. Beuth Verlag GmbH, Berlin
DIN EN ISO 8692 (2004) Wasserbeschaffenheit - Süßwasseralgen - Wachstumshemmtest mit einzelligen Grünalgen (ISO 8692). Beuth Verlag GmbH, Berlin

Eisentraeger A, Dott D, Klein J, Hahn S (2003) Comparative studies on algal toxicity testing using fluorometric microplate and Erlenmeyer flask growth-inhibitation assays. Ecotoxicol Environ Saf $54: 346-354$

Fent K (2003) Ökotoxikologie - Umweltchemie, Toxikologie, Ökologie. 2. überarb. und erw. Aufl. Georg Thieme Verlag, Stuttgart New York

Fomin A, Oehlmann J, Markert B (2003) Praktikum zur Ökotoxikologie - Grundlagen und Anwendungen biologischer Testverfahren. ecomed Verlagsgesellschaft, Landsberg

Förstner U (2000) Transferprozesse durch Sedimentresuspension. In: Guderian R, Gunkel G: Handbuch der Umweltveränderungen und Ökotoxikologie. Bd. 3b Aquatische Systeme - Biogene Belastungsfaktoren - Organische Stoffeinträge - Verhalten von Xenobiotika. Springer-Verlag, Berlin Heidelberg New York

Geis SW, Fleming KL, Korthals ET, Searle G, Reynolds L, Karner DA (2000) Modifications to the algal growth inhibitation test for use as a regulatory assay. Environ Toxicol Chem 19:36-41

HABAB-WSV (2000) Handlungsanweisung für den Umgang mit Baggergut im Binnenland. 2. überarb. Fassung. Koblenz

Henschel T, Calmano W, Maaß V, Krebs F (2003) Gefährdungsabschätzung von Gewässersedimenten - Handlungsempfehlungen und Bewertungsvorschläge für eine integrierte Bewertung. Handbuch Angewandte Limnologie (16. Erg. Lfg. 7/03) VIII-8.1. S 1-27

Hoffmann U (2000) Schnellkurs Statistik - mit Hinweisen zur SPSSBenutzung, 4. Aufl. Verlag Sport und Buch Strauß, Köln

Janssen J, Laatz W (2003) Statistische Datenanalyse mit SPSS für Windows, 4., neubearb. und erw. Aufl. Springer, Berlin

Knie J (1992) Algen-Abwassertest - Erfahrungen aus NordrheinWestfalen. In: Steinhäuser KG, Hansen P-D (Hrsg) Biologische Testverfahren. Schr.-Reihe Verein WaBoLu 89. Gustav-Fischer Verlag, Stuttgart, S 539-552

Krebs F (1988) Der pT-Wert: Ein Gewässertoxikologischer Klassifizierungs-Maßstab. GIT Fachz Lab 32:293-296

Krebs F (2000) Ökotoxikologische Bewertung von Baggergut aus Bundeswasserstrassen mithilfe der pT-Wert Methode. In: Hydrologie Wasserbewirtschaftung 44:301-307

Krebs F (2001) Ökotoxikologische Baggergutuntersuchung, Baggergutklassifizierung und Handhabungskategorien für Baggergutumlagerungen. In: Calmano W: Untersuchung und Bewertung von Sedimenten - Ökotoxikologische und chemische Testmethoden. Springer-Verlag, Berlin Heidelberg New York, S 333-352

Krebs F (2005) The pT-method as a Hazard Assessment Scheme for wastewaters. In: Blaise C, Férard J-F: Small-scale Freshwater Toxicity Investigations, vol 2. Hazard Assessment Schemes. Springer, Dordrecht, S 281-304

Lozán JL, Kausch H (2007) Angewandte Statistik für Naturwissenschaftler. 4. überarb. und erg. Aufl. Wissenschaftliche Auswertung, Berlin

Mehta CR, Patel NR, SPSS Inc. (1995) SPSS Exact Tests 6.1 for Windows. Chicago, Il

OECD (1984) Guideline for Testing of Chemicals. Alga, Growth Inhibition test. Test Guidelines No. 201. Organization for Economic Cooperation and Development. Paris

Paixão SM, Silva L, Fernandes A, O'Rourke K, Mendona E, Picado A (2008) Performance of a miniaturized algal bioassay in phytotoxicity screening. Exotoxicol 17:165-171

Pavlić Ž, Stjepanović B, Horvatić, Perśić V, Puntarić D, Ćulig J (2006) Comparative sensitivity of green algae to herbicides using erlenmeyer flask and microplate growth-inhibitation assays. Bull Environ Contam Toxicol 76:883-890

Radetski CM, Ferada JF, Blaise C (1995) A semistatic microplate-based phytotoxicity test. In: Environ Toxicol Chem 14(2):299-302 
Schmitz RPH, Ehrlichmann H, Eisenträger A, Dott W (1999) Ökotoxikologische Bewertung von Einzelstoffen und Umweltproben mit miniaturisierten aquatischen Biotestsystemen. In: Oehlmann J, Markert B: Ökotoxikologie - Ökosystemare Ansätze und Methoden. ecomed Verlagsgesellschaft, Landsberg, S 127-137
Wahrendorf D-S, Krebs F, Manz W (2005) Wirkung von AmmoniumStickstoff auf den Wachstumshemmtest mit der Grünalge Desmodesmus subspicatus nach DIN 38412 - L33. Bundesanstalt für Gewässerkunde, Koblenz, BfG-1468 